\title{
Nanometer-Scale Incoherent Imaging Using Laser-Plasma EUV Source
}

\author{
P.W. Wachulak*, A. Bartnik, H. Fiedorowicz and J. Kostecki \\ Institute of Optoelectronics, Military University of Technology, gen. S. Kaliskiego 2, 00-908 Warsaw, Poland \\ Various imaging methods and techniques capable of reaching a nanometer spatial resolution are currently \\ under development. One of them is an extreme ultraviolet microscopy, based on the Fresnel zone plates. In \\ this paper a compact, high-repetition, laser-plasma EUV source, with a gas puff target, capable of emitting \\ quasi-monochromatic radiation at $13.8 \mathrm{~nm}$ wavelength was used in the first demonstration of a desk-top EUV \\ transmission microscopy with a spatial (half-pitch) resolution of $50 \mathrm{~nm}$. EUV microscopy images of objects with \\ various thicknesses and the spatial resolution measurements using the knife-edge test are presented.
}

PACS: 52.38.Ph, 52.50.Dg, 41.50.+h, 42.30.Va, 42.79.Ci

\section{Introduction}

Imaging tools capable of resolving features on nanometer scale are currently of significant importance. Many different imaging techniques using short wavelength radiation are under development, among them being an extreme ultraviolet (EUV) and soft X-ray (SXR) microscopy, using the Fresnel zone plates [1]. New and bright short wavelength laboratory EUV sources opened a possibility for the development of table-top and desk-top microscopes that can render images of nanoscale objects with exposures as short as a few seconds and a spatial resolution approaching that of synchrotron based microscopes [2-4]. The development of bright, compact, short wavelength sources will be a significant step forward in commercialization of high resolution imaging tools in near future.

Recently different approaches for sub-micrometer resolution imaging have emerged using smaller-scale short-wavelength EUV sources such as high-order harmonics [5] and incoherent laser-plasma based sources [6]. Radiation from a capillary discharge laser, $\lambda=46.9 \mathrm{~nm}$ wavelength, was used to obtain EUV images with a spatial resolution of 120-150 nm [7]. A $13.2 \mathrm{~nm}$ wavelength radiation from Ni-like Cd EUV laser was used for imaging with a $55 \mathrm{~nm}$ resolution in reflection mode [8] and sub$-38 \mathrm{~nm}$ resolution in transmission mode [3]. Xenon based gas discharge EUV source, the Schwarzschild objective and the Fresnel zone-plate optics for second magnification step, allowed for EUV imaging reaching the spatial resolution of $\approx 100 \mathrm{~nm}$ at $\lambda=13.5 \mathrm{~nm}$ wavelength [9].

In this paper we report a compact, desk-top microscopy reaching $50 \mathrm{~nm}$ spatial resolution using a gas-

\footnotetext{
* corresponding author; e-mail: wachulak@gmail.com
}

-puff laser plasma EUV source. This type of EUV microscope utilizing short wavelength radiation from a very compact source will allow imaging of objects with high spatial resolution.

\section{Experimental setup}

The experimental arrangement is shown in Fig. 1. The laser plasma EUV source, used in the experiment, has been developed for EUV metrology applications [10] and was used in characterization of $\mathrm{Mo} / \mathrm{Si}$ multilayer mirrors [11]. This source was later modified for quasi-monochromatic emission in 13-14 nm wavelength range, as described in detail in [12]. Argon plasma was produced using laser pulses from a commercial Nd:YAG laser (Eksma), with pulse duration of $4 \mathrm{~ns}$ and energy $0.74 \mathrm{~J}$, irradiating a double stream gas-puff target. The plasma radiates in a very broad range of wavelengths, dominantly in the EUV range (5-50 $\mathrm{nm}$ wavelength). An additional spectral filtering is thus required for subsequent narrowing of the spectral emission of the source. The source can operate up to $10 \mathrm{~Hz}$ repetition rate, however, a moderate $2 \mathrm{~Hz}$ repetition rate was routinely used. A pressure of $2 \times 10^{-3}$ mbar was constantly maintained in the chamber during the source operation. The experimental setup is extremely compact. The microscope is located inside the vacuum chamber, $24 \mathrm{~cm}$ in diameter and $35 \mathrm{~cm}$ in length and the entire system fits on top of a single $2 \times 0.6 \mathrm{~m}^{2}$ optical table.

EUV radiation emitted from Ar plasma was collected, focused and spectrally filtered by an $80 \mathrm{~mm}$ in diameter, ellipsoidal, off-axis mirror coated with $\mathrm{Mo} / \mathrm{Si}$ multilayers. The mirror was corrected for the spherical aberrations. The multilayers were optimized for $13.5 \pm 0.5 \mathrm{~nm}(\mathrm{FWHM})$ wavelength range and incidence angle of 45 degrees. The theoretical reflectivity of the 


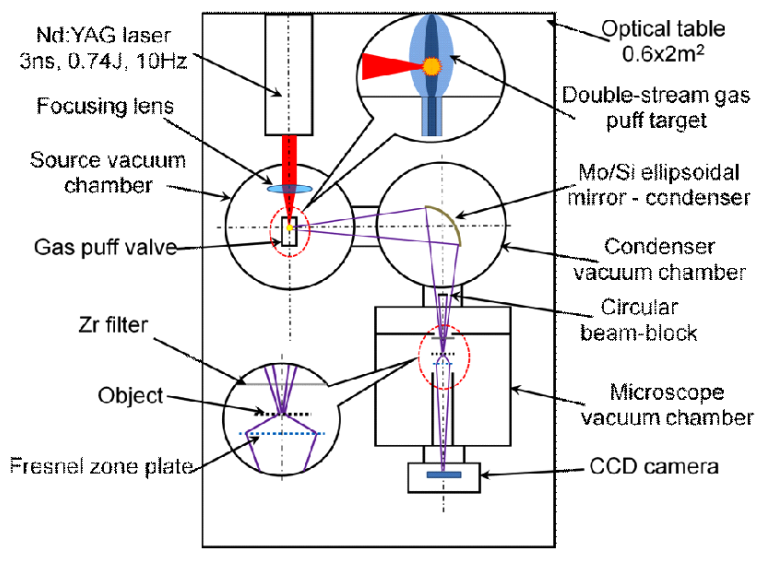

Fig. 1. An experimental arrangement of the EUV microscope (not to scale) using a laser-plasma EUV source.

mirror at $13.5 \mathrm{~nm}$ wavelength is $37.7 \%$ for an unpolarized light from the laser-plasma EUV source. The mirror produces images of the plasma with a unity lateral magnification, where both the object and image distances are equal to $254 \mathrm{~mm}$. It was developed in cooperation with Reflex s.r.o. (mirror substrate) and Fraunhofer Institut für Angewandte Optik und Feinmechanik (coating).

The laser plasma source was optimized for efficient conversion to EUV range in Ar plasma [12], where the measured in band $(\lambda=13-14 \mathrm{~nm})$ photon flux was equal to $(8.8 \pm 0.5) \times 10^{10}$ photons per pulse in a horizontally elongated spot with FWHM widths $1.09 \times 0.39 \mathrm{~mm}^{2}$, shown in Fig. 2b. That corresponds to $1.29 \mu \mathrm{J} /$ pulse. A $100 \mathrm{~nm}$ thick, $10 \mathrm{~mm}$ diameter, free-standing Zr filter (from Lebow) was used to eliminate longer wavelengths $(\lambda>18 \mathrm{~nm})$. The $\mathrm{Zr}$ filter was positioned $\approx 4-5 \mathrm{~mm}$ upstream the object. The EUV spectrum, emitted from the source, has been measured using the flat-field reflection grating spectrometer equipped with a 1200 lines/mm grating with varied groove spacing (Hitachi), $25.5 \mu \mathrm{m}$ entrance slit and $1300 \times 400$ pixels back illuminated CCD camera (Princeton Instruments) having the inverse relative bandwidth (IRB) estimated to be $\lambda / \Delta \lambda \approx 585$. The spectrum for Ar plasma in wavelength range 9-20 $\mathrm{nm}$, corrected for Zr filter transmission, both direct and spectrally filtered by condenser optic is shown in Fig. 2a. This quasi-monochromatic spectrum has two main spectral peaks, namely $2 p^{6} 3 p-2 p^{6} 5 d$ at $13.793 \mathrm{~nm}$ and $2 p^{6} 3 s-2 p^{6} 5 p$ at $13.844 \mathrm{~nm}$ transitions in $\mathrm{Ar}^{\mathrm{VIII}}$ [13]. The IRB (FWHM) of filtered spectrum is $\lambda / \Delta \lambda=140$ at $\lambda=13.84 \mathrm{~nm}$, however, can be underestimated due to relatively low spectral resolution of our spectrometer $(0.24 \AA)$.

Two different objects, with different thicknesses were used. First one is a copper G2000HS Fine Square Mesh (SPI Supplies) with $12.5 \mu \mathrm{m}$ period, $5 \mu \mathrm{m}$ width bar and $4 \mu \mathrm{m}$ thick $(\approx 11 \times$ DOF — depth of focus $)$. The second one is a Quantifoil holey $300 \mathrm{M}$ carbon foil supported on a steel mesh (SPI supplies), $10 \mathrm{~nm}$ thick according to man-

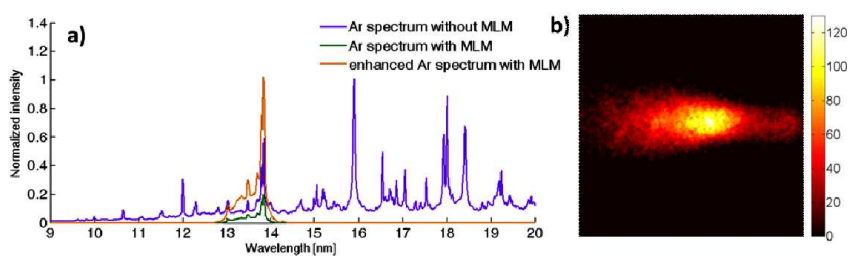

Fig. 2. Spectrum of Ar plasma radiation (a), intensity distribution illuminating the object (b) (size in the text).

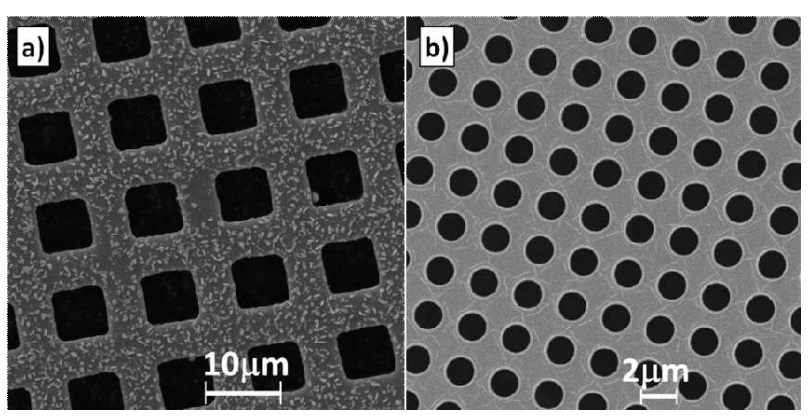

Fig. 3. SEM images of two objects imaged with EUV microscope: a copper mesh, $4 \mu \mathrm{m}$ thick (a) and perforated carbon foil, coated additionally by a thin layer of gold, total thickness of $70 \mathrm{~nm}$ (b).

ufacturer's specifications, having $1.5 \mu \mathrm{m}$ diameter holes spaced on a square grid with $2.5 \mu \mathrm{m}$ period. To improve the optical contrast carbon foil was additionally coated with $\approx 60 \mathrm{~nm}$ thick layer of gold, having a transmission of $4.3 \%$ at $13.84 \mathrm{~nm}$ wavelength, thus in total having thickness of $\approx 70 \mathrm{~nm}(0.2 \times$ DOF $)$. Typical scanning electron microscopy (SEM) images of the mesh and the perforated foil are shown in Fig. 3a and b, respectively.

The objects, placed $254 \mathrm{~mm}$ from the mirror, are imaged using a Fresnel zone plate (ZP) objective onto EUV sensitive CCD camera iKon-M (Andor) with $1024 \times 1024$ pixels and $13 \times 13 \mu \mathrm{m}^{2}$ pixel size. The $\mathrm{ZP}$ was fabricated by Zone Plates Ltd., using electron beam lithography in $220 \mathrm{~nm}$ thick poly(methyl methacrylate) layer spin coated on top of a $50 \mathrm{~nm}$ thick silicon nitride $\mathrm{Si}_{3} \mathrm{~N}_{4}$ membrane. ZP diameter is equal to $D=200 \mu \mathrm{m}$, number of zones $N_{\mathrm{ZP}}=1000$ and an outer zone width $\Delta r=50 \mathrm{~nm}$. The ZP was fabricated and optimized for $\lambda=13.5 \mathrm{~nm}$, but it was used with a quasi-monochromatic illumination around $\lambda=13.84 \mathrm{~nm}$ from Ar plasma. The ZP focal length was $f=722.5 \mu \mathrm{m}$ and the numerical aperture was equal to $\mathrm{NA}=0.138$. The DOF, calculated as $\mathrm{DOF}= \pm \lambda /\left(2 \mathrm{NA}^{2}\right)$ was equal to $\pm 363.4 \mathrm{~nm}$. For both objects the magnification, ranging from $520-840 \times$, was used, adjusted by changing the camera to ZP distance and refocusing for "sharpest" image. More information regarding the EUV microscope can be found in $[4,14]$.

Geometrical numerical apertures of the collecting ellipsoidal mirror in horizontal and vertical directions are equal to $\mathrm{NA}_{\mathrm{cH}}=0.11$ and $\mathrm{NA}_{\mathrm{cV}}=0.15$, respec- 
tively, and are matched to the numerical aperture of the $\mathrm{ZP}, \mathrm{NA}_{\mathrm{ZP}}=0.138$, thus providing incoherent illumination [15], since $\sigma_{\mathrm{H}, \mathrm{V}}=\mathrm{NA}_{\mathrm{cH}, \mathrm{V}} / \mathrm{NA}_{\mathrm{ZP}}$ equal to $\left[\sigma_{\mathrm{H}}, \sigma_{\mathrm{V}}\right]=[0.8,1.1]$. The $\mathrm{ZP}$ was mounted on three axis motorized translation stage (Standa). A piezoelectric, $25 \mu \mathrm{m}$ travel, single axis flexure stage, model NF15A (Thorlabs), was used to adjust precisely the object-ZP distance at a rate of $3 \mathrm{~V} / \mu \mathrm{m}$ with theoretical resolution reaching $10 \mathrm{~nm}$. To provide a conical illumination of the object and avoid stray light through the ZP a circular beam block, $12 \mathrm{~mm}$ in diameter, was placed $\approx 15 \mathrm{~cm}$ from the ZP. The required exposure in case of the mesh object and foil was 50 and 100 EUV pulses at $2 \mathrm{~Hz}$ repetition rate, respectively. The source can operate at up to $10 \mathrm{~Hz}$ repetition rate, however, the pressure buildup in the microscope chamber might cause re-absorption of EUV photons in neutral gas. The CCD camera was cooled down to $-20{ }^{\circ} \mathrm{C}$ to decrease intrinsic noise during the image acquisition.

\section{Experimental results and discussion}

For each object the microscope alignment was optimized to provide a uniform illumination in the entire field of view, also, during the image acquisition, the object$\mathrm{ZP}$ distance was changed using the piezoelectric stage by $\Delta z \approx 330 \mathrm{~nm}$, corresponding to $1 \mathrm{~V} /$ step, to obtain the sharpest possible EUV image, over the $z$-range of $\approx \pm 20 \mu \mathrm{m}$ from the $\mathrm{ZP}$ focal point. The $\Delta z$ was chosen to be smaller than DOF. From the entire set of images, for each object, the "sharpest" EUV image was chosen for subsequent resolution measurements. Resolution of the microscope was assessed by a well established knife edge (KE) test. For incoherent illumination the 10-90\% intensity transition across a sharp edge corresponds to a well known Rayleigh resolution and to twice the value of half-pitch grating resolution of the optical system [1, 15].

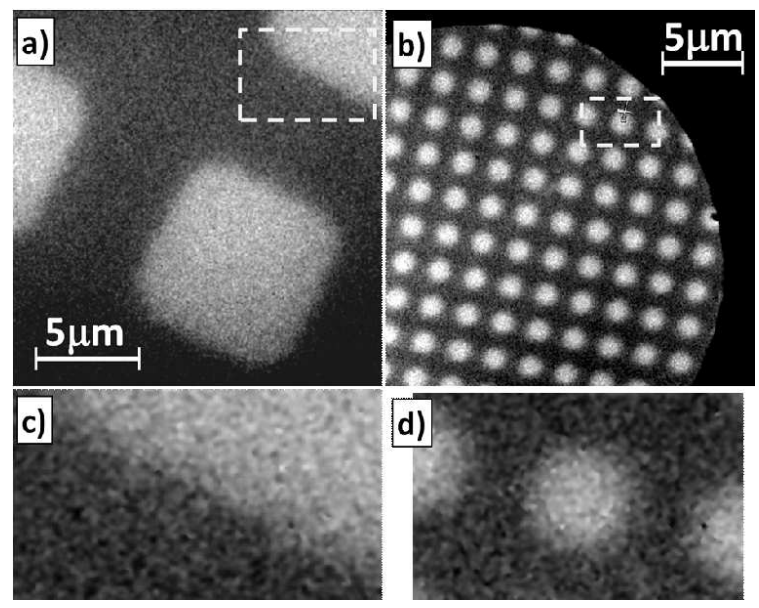

Fig. 4. EUV images of $\mathrm{Cu}$ mesh object (a, c) and foil (b, d) with Ar plasma illumination. (c, d) are the magnified subsections of larger EUV images indicated by boxed regions.
Typical EUV images of the mesh object (a, c) and the perforated foil object $(b, d)$ under illumination by $\mathrm{Ar}$ plasma radiation are depicted in Fig. 4, where the boxed regions show where subsequent KE resolution measurements were carried out. The results of half-pitch KE measurements were based on 6 independent KE measurements for each object and obtained based on period of imaged structure for higher accuracy. Lineouts across EUV images were assessed by averaging 5 adjacent lines in the EUV image to improve a signal to noise ratio. The best KE half-pitch resolution was measured to be $51.0 \pm 10.6 \mathrm{~nm}$ for thin, perforated foil object illuminated by quasi-monochromatic radiation from Ar plasma. Slightly worse resolution of $72.7 \pm 5.0 \mathrm{~nm}$ was obtained for thicker mesh object, due to its thickness of $\approx 11 \times$ DOF.

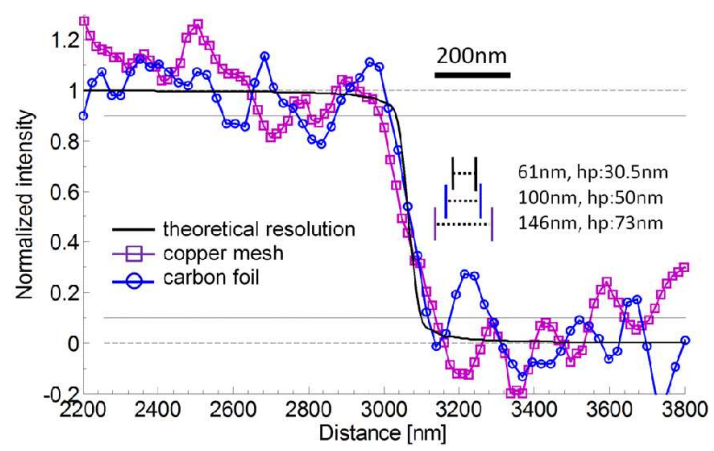

Fig. 5. Typical KE lineouts indicating 10-90\% intensity transition in the EUV image related to the Rayleigh resolution criterion for both objects and the theoretical KE limit.

Typical KE lineouts, obtained in boxed regions, for the images in Fig. 4, for each object and the theoretical $\mathrm{KE}$ resolution limited by $\mathrm{ZP}$ outer zone width $\Delta r$, are depicted in Fig. 5. Figure shows also the full (10-90\%) and half-pitch Rayleigh resolution measurements.

The theoretical half-pitch resolution of the microscope can be expressed as $r_{\mathrm{KE}}=k \lambda /\left(2 \mathrm{NA}_{\mathrm{ZP}}\right)=k \Delta r$, where $k$ is illumination dependent and resolution test specific constant [15], NA $\mathrm{ZP}_{\mathrm{P}}$ is the numerical aperture of the objective $\mathrm{ZP}$, for incoherent illumination $(k=0.61)$ this resolution is equal to $30.5 \mathrm{~nm}$. The theoretical resolution can only be achieved for "thin" objects and monochromatic radiation, when the monochromaticity criterion $\lambda / \Delta \lambda>$ $N_{\mathrm{ZP}}=1000$ will be fulfilled. For quasi-monochromatic Ar plasma emission, $\lambda / \Delta \lambda=140<N_{\mathrm{ZP}}$, the resolution is worse, equal to $51 \mathrm{~nm}$ (for the foil). If the object is much thicker than the DOF, $t=11 \times \mathrm{DOF}$ for mesh, measured resolution is $72.7 \mathrm{~nm}$. This change in resolution can be attributed to the significant object thickness.

\section{Conclusions}

In summary, we have demonstrated near $50 \mathrm{~nm}$ spatial resolution desk-top EUV transmission microscope based on a Fresnel diffractive optics and a gas-puff, laser-plasma 
EUV source. This desk-top microscope, under the incoherent illumination from Ar plasma, allows to capture images at $13.8 \mathrm{~nm}$ wavelength with the spatial resolution of $51 \mathrm{~nm}$ and the exposure time of $50 \mathrm{~s}$, comparable to larger table-top and synchrotron-based systems.

\section{Acknowledgments}

The research was supported by the Foundation for Polish Science under the HOMING 2009 Programme (grant number HOM2009/14B), the EC's 7. Framework Program (LASERLAB-EUROPE - grant agreement no. 228334 and COST Action MP0601, also co-funded by Polish Ministry of Science and Education, decision number 816/N-COST/2010/0), European Economic Area (EEA) Grants. We would like to thank to Dr. R. Jarocki, Dr. R. Rakowski, Dr. M. Szczurek and A. Szczurek, M. Sc., for their contribution to the development of the EUV source, to Prof. L. Pina and Dr. T. Feigl, for fabrication of the multilayer condenser optic, to Prof. M.C. Marconi and Prof. C.S. Menoni for the constructive discussions and to Michaela Martinkova for her comments and participation in the experiment during her EUV Training Programme at MUT.

\section{References}

[1] D. Attwood, Soft X-rays and Extreme Ultraviolet Radiation, Cambridge University Press, Cambridge 1999.

[2] P.A.C. Takman, H. Stollberg, G.A. Johansson, A. Holmberg, M. Lindblom, H.M. Hertz, J. Microsc. 226, 175 (2007).

[3] G. Vaschenko, C. Brewer, F. Brizuela, Y. Wang, M.A. Larotonda, B.M. Luther, M.C. Marconi, J.J. Rocca, C.S. Menoni, Opt. Lett. 31, 1214 (2006).

[4] P.W. Wachulak, A. Bartnik, H. Fiedorowicz, Opt. Lett. 35, 2337 (2010).
[5] M. Wieland, C. Spielmann, U. Kleineberg, T. Westerwalbesloh, U. Heinzmann, T. Wilhein, Ultramicroscopy 102, 93 (2005).

[6] I.A. Artioukov, A.V. Vinogradov, V.E. Asadchikov, Y.S. Kasyanov, R.V. Serov, A.I. Fedorenko, V.V. Kondratenko, S.A. Yulin, Opt. Lett. 20, 2451 (1995).

[7] G. Vaschenko, F. Brizuela, C. Brewer, M. Grisham, H. Mancini, C.S. Menoni, M.C. Marconi, J.J. Rocca, W. Chao, J.A. Liddle, E.H. Anderson, D.T. Attwood, A.V. Vinogradov, I.A. Artioukov, Y.P. Pershyn, V.V. Kondratenko, Opt. Lett. 30, 2095 (2005).

[8] F. Brizuela, Y. Wang, C.A. Brewer, F. Pedaci, W. Chao, E.H. Anderson, Y. Liu, K.A. Goldberg, P. Naulleau, P. Wachulak, M.C. Marconi, D.T. Attwood, J.J. Rocca, C.S. Menoni, Opt. Lett. 34, 27 (2009).

[9] L. Juschkin, R. Freiberger, K. Bergman, J. Phys., Conf. Ser. 186, 012030 (2009).

[10] H. Fiedorowicz, A. Bartnik, R. Jarocki, J. Kostecki, J. Krzywinski, J. Mikołajczyk, R. Rakowski, A. Szczurek, M. Szczurek, J. Alloys Comp. 401, 99 (2005).

[11] R. Rakowski, A. Bartnik, H. Fiedorowicz, R. Jarocki, J. Kostecki, J. Krzywiński, J. Mikołajczyk, L. Pina, L. Ryć, M. Szczurek, H. Ticha, P. Wachulak, Opt. Appl. 36, 4 (2006)

[12] P.W. Wachulak, A. Bartnik, H. Fiedorowicz, T. Feigl, R. Jarocki, J. Kostecki, R. Rakowski, P. Rudawski, M. Sawicka, M. Szczurek, A. Szczurek, Z. Zawadzki, Appl. Phys. B 100, 461 (2010).

[13] R.L. Kelly, J. Phys. Chem. Ref. Data 16 supplement 1, 402 (1987).

[14] P.W. Wachulak, A. Bartnik, H. Fiedorowicz, J. Kostecki, Opt. Exp. 19, 9541 (2011).

[15] J.M. Heck, D.T. Attwood, W. Meyer-Ilse, E.H. Anderson, J. X-Ray Sci. Technol. 8, 95 (1998). 